\title{
COMPOSITION OPERATORS ISOLATED IN THE UNIFORM OPERATOR TOPOLOGY
}

\author{
EARL BERKSON ${ }^{1}$
}

\begin{abstract}
It is is shown that $\phi$ is an analytic map of the disc $|z|<1$ into itself such that $\phi$ has radial limits of modulus 1 on a set of positive measure, then for $1<p<\infty$ the corresponding composition operator on $H^{p}$ is isolated in the topological space of composition operators on $H^{p}$ (with the uniform operator topology).
\end{abstract}

In this note we show that a composition operator whose composing function has a boundary function of modulus 1 on a set of positive measure is isolated in the metric space of composition operators (the metric being distance in the space of bounded operators). Throughout what follows, let $K$ be the unit circle $|z|=1$ in the complex plane C. Let $D=\{z \in \mathbf{C}:|z|<1\}$. We denote normalized Lebesgue measure on $K$ by $\sigma$. For a function $f$ in $H^{\infty}(D)$ we denote its boundary function by $\tilde{f}$. As a convenience, we shall standardize $\tilde{f}$ as the radial limit of $f$ where the radial limit exists, and as 0 where the radial limit does not exist.

THEOREM. Let $\phi$ be an analytic map of $D$ into $D$ such that $\sigma(A)>0$, where $A=\tilde{\phi}^{-1}(K)$. Let $\psi$ be an analytic map of $D$ into $D$, and let $C_{\phi}, C_{\psi}$ be the corresponding composition operators on $H^{p}(D), 1<p<\infty$. If $\left\|C_{\psi}-C_{\phi}\right\|<$ $[\sigma(A) / 2]^{1 / p}$, then $\psi=\phi$.

Proof. Let $B=\tilde{\psi}^{-1}(K)$. For each positive integer $n$,

$$
\left.\int_{A \backslash B}|| \tilde{\psi}\right|^{n}-\left.1\right|^{p} d \sigma<\int_{K}\left|\tilde{\psi}^{n}-\tilde{\phi}^{n}\right|^{p} d \sigma<\left\|C_{\psi}-C_{\phi}\right\|^{p}
$$

Letting $n \rightarrow \infty$, we get, by bounded convergence, that $\sigma(A \backslash B)<\left\|C_{\psi}-C_{\phi}\right\|^{p}<$ $\sigma(A) / 2$. Hence

$$
\sigma(A \cap B)>\sigma(A) / 2 .
$$

Let $J$ be an arc on $K$, and let $E$ be the set consisting of the endpoints of $J$ ( $J$ may exclude one or both points of $E$ ). By (1) neither $\phi$ nor $\psi$ is constant, and hence $\sigma\left(\tilde{\psi}^{-1}(E)\right)=\sigma\left(\tilde{\phi}^{-1}(E)\right)=0$. For each positive integer $n$, define $k_{n}$ on $K$ by taking $k_{n}$ to be identically 1 on $J$ and identically $n^{-1}$ on $K \backslash J$. Let $F_{n}$ be the (bounded) outer

Received by the editors January 10, 1980. Presented to the Society, August 21, 1980 at the Special Session on Hardy Spaces and Harmonic Analysis.

AMS (MOS) subject classifications (1970). Primary 30A78, 47B99.

Key words and phrases. Composition operator, $H^{p}$.

${ }^{1}$ The work of the author was supported by a National Science Foundation grant. 
function on $D$ induced by $k_{n}$ :

$$
F_{n}(z)=\exp \left\{(2 \pi)^{-1} \int_{0}^{2 \pi}\left(e^{i t}+z\right)\left(e^{i t}-z\right)^{-1} \log k_{n}\left(e^{i t}\right) d t\right\}
$$

Since $k_{n}$ is continuous on $K \backslash E$, a standard argument using a theorem of Lindelöf [3, Theorem 4, p. 346] shows that there is a subset $W_{n}$ of $A \cap B$ such that $\sigma\left(W_{n}\right)=0$, and, on $(A \cap B) \backslash W_{n},\left|\widetilde{C_{\psi}\left(F_{n}\right)}\right|=k_{n}(\tilde{\psi}),\left|\widetilde{C_{\phi}\left(F_{n}\right)}\right|=k_{n}(\tilde{\phi})$. For all $n$,

$$
\int_{(A \cap B) \backslash W_{n}}\left|k_{n}(\tilde{\psi})-k_{n}(\tilde{\phi})\right|^{p} d \sigma<\left\|C_{\psi}-C_{\phi}\right\|^{p}\left\|F_{n}\right\|_{p}^{p} \text {. }
$$

Noting that $\sigma\left(W_{n}\right)=0$, and using the definition of $F_{n}$, we can replace this inequality by

$$
\int_{A \cap B}\left|k_{n}(\tilde{\psi})-k_{n}(\tilde{\phi})\right|^{p} d \sigma \leqslant\left\|C_{\psi}-C_{\phi}\right\|^{p}\left\{\sigma(J)+n^{-p} \sigma(K \backslash J)\right\} .
$$

Denote by $k_{J}$ the characteristic function (on $K$ ) of $J$. Let $n \rightarrow \infty$ in the last inequality and get

$$
\int_{A \cap B}\left|k_{J}(\tilde{\psi})-k_{J}(\tilde{\phi})\right|^{p} d \sigma<\left\|C_{\psi}-C_{\phi}\right\|^{p} \sigma(J)
$$

Let $\Psi$ and $\Phi$ be, respectively, the restrictions to $A \cap B$ of $\tilde{\psi}$ and $\tilde{\phi}$. Using the symbol $\Delta$ to indicate the symmetric difference of sets, we can now restate (2) as

$$
\sigma\left(\Psi^{-1}(J) \Delta \Phi^{-1}(J)\right) \leqslant\left\|C_{\psi}-C_{\phi}\right\|^{p} \sigma(J)
$$

for any $\operatorname{arc} J$ on $K$. For each positive integer $n$, partition $K$ into finitely many disjoint $\operatorname{arcs} J_{1}^{(n)}, J_{2}^{(n)}, \ldots, J_{m_{n}}^{(n)}$, each of length less than $n^{-1}$. Let

$$
Q_{n}=\bigcup_{k=1}^{m_{n}} \Psi^{-1}\left(J_{k}^{(n)}\right) \Delta \Phi^{-1}\left(J_{k}^{(n)}\right) \text {. }
$$

On $(A \cap B) \backslash Q_{n}$ we have

$$
|\Psi-\Phi|<n^{-1}
$$

Set $Q=\lim \inf _{n} Q_{n}$. Then $\sigma(Q) \leqslant \liminf _{n} \sigma\left(Q_{n}\right)$. By (3), $\sigma\left(Q_{n}\right)<\left\|C_{\psi}-C_{\phi}\right\|^{p}$ for all $n$. So $\sigma(Q) \leqslant\left\|C_{\psi}-C_{\phi}\right\|^{p}<\sigma(A) / 2$. By $(1), \sigma((A \cap B) \backslash Q)>0$. However, by (4), $\Psi=\Phi$ on $(A \cap B) \backslash Q$. By [2, Theorem (2.2)] $\psi=\phi$.

By virtue of Remark (iii) we have

COROLlaRY. Let $\phi$ and $\psi$ be nonconstant inner functions, and let $C_{\phi}$, $C_{\psi}$ be the corresponding composition operators on $H^{p}(D), 1<p<\infty$. If $\left\|C_{\phi}-C_{\psi}\right\|<1$, then $\phi=\psi$.

REMARKs. (i) The metric space of composition operators on $H^{p}(D)$ (with metric the norm-distance between operators) is clearly not discrete. In fact, if $\phi$ is an analytic map of $D$ into a compact subset of $D$, then it is easy to see that $\lim _{r \rightarrow 1^{-}}\left\|C_{r \phi}-C_{\phi}\right\|=0$.

(ii) In [1] it was shown that for $1<p<\infty$, the identity operator is isolated in the space of composition operators on $H^{P}(D)$ (with the uniform operator topology). The theorem above extends this result. 
(iii) The Corollary can be obtained by using the method of proof of the Theorem with obvious modifications such as using $K$ instead of the sets $A$ and $B$. However, the author is indebted to the referee for the following more general observation. By dispensing with the use of (1) the proof of the Theorem shows the following proposition: if $\phi$ and $\psi$ are analytic maps of $D$ into $D, A$ and $B$ are, respectively, $\tilde{\phi}^{-1}(K)$ and $\tilde{\psi}^{-1}(K)$, and $1<p<\infty$, then $\left\|C_{\phi}-C_{\psi}\right\|<[\sigma(A \cap B)]^{1 / p}$ implies $\phi=\psi$. The Corollary then follows directly from the statement of this proposition. Moreover, the Theorem itself is a consequence of this proposition. One need only obtain (1) as before.

\section{REFERENCES}

1. E. Berkson and H. Porta, The group of isometries on Hardy spaces of the n-ball and the polydisc, Glasgow Math. J. (to appear).

2. P. Duren, Theory of $H^{p}$ spaces, Pure and Appl. Math., Vol. 38, Academic Press, New York and London, 1970.

3. G. M. Goluzin, Geometric theory of functions of a complex variable, Transl. Math. Monos., Vol. 26, Amer. Math. Soc., Providence, R.I., 1969.

Department of MAThematics, Universtty of IllinoIs, Urbana, ILlinoIs 61801 